\title{
EARTH OBSERVATION APPLICATIONS FOR GOAL 14: IMPROVING MARITIME DOMAIN AWARENESS USING SYNTHETIC APERTURE RADAR IMAGING WITH AUTOMATIC IDENTIFICATION SYSTEM IN THE PHILIPPINES
}

\author{
R. Vicente 1, L. Tabanggay 1, J. Rayo1, K. Mina 1, A. Retamar 1 \\ 1 Department of Science and Technology - Advanced Science and Technology Institute, Diliman, Quezon City, Philippines 1101 - \\ (rocellvicente, lianne.tabanggay, joshua.rayo, katrina.mina, ning)@asti.dost.gov.ph
}

KEY WORDS: Earth observation, maritime domain awareness, Sustainable Development Goal 14: Life Below Water, synthetic aperture radar, automatic identification system

\begin{abstract}
:
The Philippines has acquired access to the NovaSAR-1 satellite developed by Surrey Satellite Technology, Ltd. (SSTL) for the implementation of its project Synthetic Aperture Radar (SAR) and Automatic Identification System (AIS) for Innovative Terrestrial Monitoring and Maritime Surveillance, which will provide simultaneous S-band SAR imaging with AIS data for applications targeted on improving maritime domain awareness. The country has inherent challenges in this field due to its archipelagic nature, with earth observation seen as a potential solution as it provides an immediate and wide coverage over designated priority areas. This contributes toward achieving Sustainable Development Goal 14: Life Below Water by providing objective information in support of data-driven decision and policymaking, closing knowledge gaps in monitoring Philippine waters.
\end{abstract}

\section{INTRODUCTION}

The Department of Science and Technology - Advanced Science and Technology Institute (DOST-ASTI) in the Philippines is implementing the Synthetic Aperture Radar (SAR) and Automatic Identification System (AIS) for Innovative Terrestrial Monitoring and Maritime Surveillance, otherwise known as the SAR with AIS project, to improve overall maritime domain awareness (MDA) in the country. One of the core resources of the project is Surrey Satellite Technology, Ltd.'s (SSTL) NovaSAR-1 satellite which allows simultaneous S-band SAR imaging with concurrent AIS data. Being a mission partner, the country has access to a $10 \%$ share of the available imaging capacity of the satellite, further increasing capabilities in earth observation over Philippine waters. This project was conceptualized and implemented in response to coordinated inputs from local maritime monitoring agencies on the inherent challenges in policing an archipelago.

\subsection{Rationale}

The Philippines shares sea borders with China, Japan, Taiwan, Malaysia, Vietnam, Indonesia and Palau, with country observing economic growth from the available livelihoods supported by maritime activities in these seven states. However, the constant traffic across island ports pose issues in vessel detection and monitoring due to limitations brought by the lack of available technology and resources, enforcement, regulation and policies on maritime security and domain awareness.

Among the United Nations (UN) Sustainable Development Goals (SDGs)is Goal 14: Life Below Water, which aims to conserve and promote sustainable growth and development of the blue economy. One of the country's policies that are in line with Goal 14 is the Philippine Fisheries Code of 1998 (Republic Act No. 8550 or RA 8550 ) that supports not only the livelihood of fisherfolk but regular transactions that partake on the Philippine seas such as transportation, tourism, and freight.

In concurrence with RA 8550, the Fisheries Administrative Order No. 260 Series of 2018 (FAO 260 S 2018) regulates the Philippines' usage of vessel monitoring measures (VMM) and electronic reporting systems (ERS) for commercial vessels and aims for its complete implementation by 2020 . Some examples of VMMs include Automatic Location Communicators (ALC), Mobile Tracking-Transceiver Units (MTU), and Automatic Identification System (AIS).

Regulatory bodies and agencies rely on available AIS monitoring and detection technology for the enforcement of these laws and subsequent violations. While current implementation of various VMMs with respective terrestrial transponders is ongoing, there are challenges in mobilizing and utilizing this technology due to possible tampering issues. In the absence of a redundancy measure, this may become a concern for maritime domain awareness in the Philippines.

\subsection{Review of Related Literature}

1.2.1 Goal 14: Life Below Water places importance on the conservation, protection and sustainable development of oceans, seas and marine resources (UN, 2016). These play key roles in climate change mitigation and adaptation alongside providing vital natural resources such as food, medicine, biofuels and other products. Moreover, more than 3 billion people all over the world depend on marine and coastal biodiversity for their livelihood, further highlighting the importance of achieving this goal (UN, 2017).

One of the biggest challenges globally is creating a viable means of integrating social, economic and political importance over maritime domains while providing communities visibility over 
micro-level challenges such as security, access to clean waters and equity of access to these waters (Anderson et.al, 2017). Among the targets of Goal 14 which earth observation (EO) can play a vital role is in (1) effective regulation of harvesting overfishing, (2) conservation of at least $10 \%$ of coastal and marine areas consistent with national and international law, and (3) enhancement of the conservation and sustainable use of oceans and their resources by implementing international laws as reflected in UNCLOS (Palomino, 2020).

Achieving (1) and (2) pose certain challenges particularly in an archipelagic country such as the Philippines with more than 36,000 kilometers $(\mathrm{km})$ of coast to monitor alongside its 2.2 million $\mathrm{km} 2$ exclusive economic zone (EEZ) (Garcia, 2005). The Philippines roughly has around 680,000 square $\mathrm{km}$ of total marine resources with abundant biodiversity and ecosystems that also serve as the livelihood of coastal communities and other neighboring countries.

Effective conservation and sustainable use of the ocean and the associated resources with it hinges on the implementation and compliance to international law (3). This, however, may be difficult to police and monitor, given the aforementioned challenges in archipelagos with a vast maritime domain. Mismanagement of this key resource can result to loss of economic benefits in the fishing sector alone amounting to US $\$ 50 \mathrm{~B}$ annually, with an overall economic impact of US\$200B per year (UN, 2017).

1.2.2 Earth Observation Applications related to Goal 14. The success of (1), (2), and (3) is dependent on each country's capabilities in the field of MDA. EO can support applications for MDA by providing timely, objective information over specific areas of interest, supporting data-driven assessment and decisionmaking for governments (Prado et.al., 2019). Multi-disciplinary research has the potential to bridge existing knowledge gaps by bringing different disciplinary knowledge together, such as the case for merging remote sensing with policy and enforcement (Sturesson, Weitz, and Persson, 2018, p.7).

The effectiveness of (1), (2), and (3) hinges not only on existing policies but also on the amount of data available to them. Challenges in obtaining data for MDA exist partly due to the need to monitor a wide area with a short turnaround time. EO is a possible solution for this, particularly SAR imaging, where surveillance can be established regardless of weather conditions. However, the accessibility of EO data and other subsequent datasets does not suffice to fully achieve the SDGs, as these data should be operated to be made as productive as possible for units contributing to the welfare of the SDGs (Anderson et.al., 2019).

1.2.3 SAR for Ship Detection and Monitoring remains beneficial for projects where overall surveillance and domain awareness are crucial. SAR imaging has been used for ship and vessel detection as the signatures of marine structures show high reflectance in images in contrast with bodies of water.

Pichel, Clemente-Colón, Wackerman, and Friedman (2018) pose five limits of SAR-based ship detection: "ship characteristics, environmental conditions, radar characteristics, image quality, and image resolution." Variations in these categories can greatly affect the SAR image capture and post-processing, as there might be tradeoffs with high spatial coverage and image resolution (Meyer and Hinz, 2009).

Relying on visual inspection alone may not be adequate for vessel monitoring and enforcement of maritime rules and regulations, as these may be circumvented by disabling the transmission of AIS data. A possible solution is through SAR-based vessel detection, as SAR images can detect marine vessels blocking AIS transmission.

However, without the consideration of available AIS data, ship detection solely using SAR data creates difficulty in segregation, identification and detection in busy maritime regions (Chaturvedi, Yang, Ouchi, and Shanmugam, 2012, p.324). Through AIS, ships transmit its information to surrounding ships and to nearby terrestrial AIS transponders. Furthermore, dynamic information, such as the ship's position, speed and heading, automatically generates updates every 2 to 10 seconds while static information, such as the ID, type and size is updated every 6 minutes (Achiri, Guida, \& Iervolino, 2018, p. 1). These data may be used to produce reliable and predictable results in support of the development of automated detection systems for monitoring maritime activity (Meyer and Hinz, 2009).

\section{MAIN BODY}

\subsection{EO Opportunity}

An assessment of the indicators of Goal 14 shows that remote sensing can be beneficial in providing reliable information for the monitoring and implementation of the goal (Virto, 2018). According to Virto, the EO sector can directly help in providing information for the Goal 14 targets (2018). These targets include 14.5 that focuses on the conservation of coastal and marine areas and target 14.6 that aims to eliminate types of fishing that can possibly harm the marine economy. According to the United Nations Office for Outer Space Affairs (UNOOSA), the space sector can also support Goal 14 by:

- mapping and monitoring of natural and protected areas,

- fishing vessel tracking and navigation,

- monitoring of illegal, unregulated and unreported fisheries, among others (2020).

In the Philippines, space technology, such as data from EO satellites, has been used for disaster risk reduction and management and environmental monitoring. In 2016, the country launched its very own microsatellite tasked to gather spaceborne data for scientific EO. Since then, other space infrastructure were put in place to cater to the growing demand for space-based data.

Among these is the launch of the SAR with AIS project in 2018, strengthening the country's capability to monitor infrastructure in offshore islands and marine areas alongside detecting ships within the Philippines.

2.1.1 SAR with AIS Project. The DOST-ASTI's SAR with AIS project aims (a) to operationalize the distribution of SAR and AIS data for the Philippine government and (b) to conduct research on applications for terrestrial monitoring and maritime surveillance. The project will primarily leverage data from the 
NovaSAR-1 satellite, which can cover up to 81,600 sq.km per day at $20 \mathrm{~m}$ resolution using its ScanSAR mode with the country's existing imaging share. Among its deliverables is an automated ship detection and structure identification system that can be used to track ships with AIS and detect those that do not transmit AIS data.

The use of AIS has evolved to become a tool for close monitoring of ship route anomalies, such as ship presence in restricted areas (Papi et.al., 2014). With the SAR with AIS project in operation, the Philippines could be able to effectively monitor ships, both foreign and local, that are within its maritime domain, identify ships that are inside restricted waters, as well as track infrastructure along coastal and marine areas.

As displayed in Figure 1, the SAR with AIS Project generated a georeferenced and corrected SAR image of Batangas City Port, Batangas, Philippines. From visual inspection, the small speckles scattered around coastlines are assumed to be marine vessels.

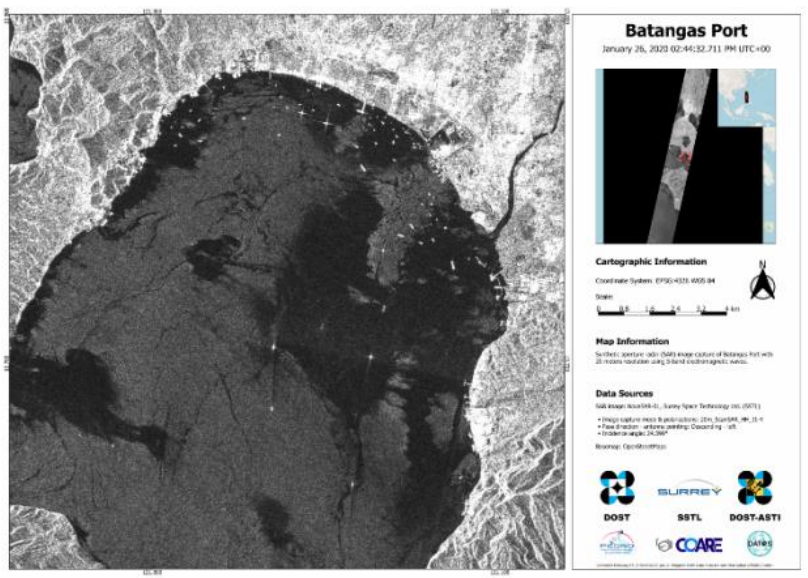

Figure 1. SAR image capture of Batangas City Port using ScanSAR mode, 2019 @ SSTL.

The above data, among many others, enable visual inspection for maritime management agencies to devise innovative traffic regulation and port security measures.

2.1.2 The SIYASAT Portal. The data from the NovaSAR1 satellite is further processed and applied to several EO applications that prioritize the needs of the project partners. The Surveillance, Identification, and Assessment Using Satellites (SIYASAT) Portal is a home-grown image collection and planning system for the stakeholders of the SAR with AIS project (siyasat means 'to investigate' in Filipino). The SIYASAT Portal enables its users to task the NovaSAR-1 satellite and create image requests suited to their requirements.

Figure 2.a. shows the interface of the SIYASAT Portal Planning and Tasking module as made available to the project partners.

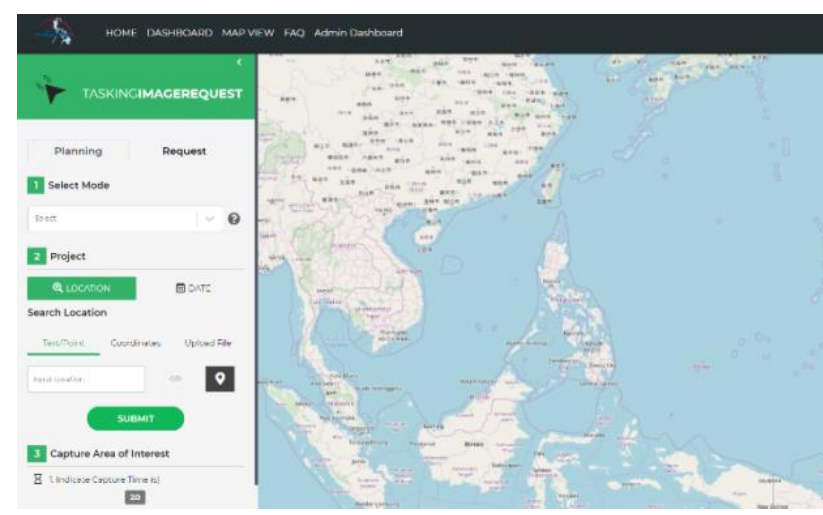

Figure 2.a. Tasking and planning module in the SIYASAT Portal.

End-users can generate pass projections of the NovaSAR-1 portal across their areas of interest (AOIs) for the system's maximum period of seven (7) days from the date of request. The users can carefully plan and allocate their imaging requirements and submit multiple requests within the period. DOST-ASTI conducts documentation, deconfliction and approval of these requests, as shown in Figure 2.b.

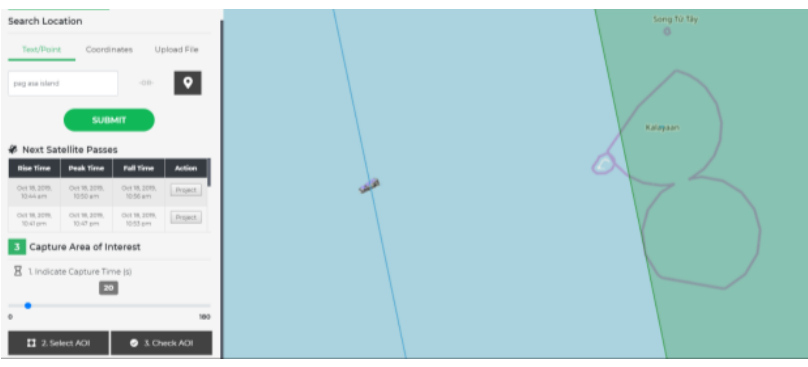

Figure 2.b. Projection of the right-looking projection of the NovaSAR-1 satellite in 30m ScanSAR mode.

The SIYASAT Portal provides an avenue for stakeholders to access both raw and processed concurrent SAR with AIS data for subsequent decision-making and policy implementation and will be expanded to become a full-fledged maritime surveillance and monitoring system.

2.2 AIS Data. Satellite-based AIS can capture signals from remote areas where terrestrial AIS receivers are unavailable for installation. The AIS payload on NovaSAR-1 uses a conventional non-coherent receiver for on-board processing. AIS signals sent from ships are collected randomly, decoded directly on the satellite and stored to its memory for downlink on a ground station (exactEarth, 2015). However, not all packets can be captured from high-traffic areas and signals are also subject to time slot collision. Satellite-based AIS enables policymakers to better understand ship activity on large water bodies and enforce regulations to monitor maritime activities.

2.3 Automatic Ship Detection. An automatic ship detection algorithm was developed with this system to identify ships as detected from SAR images. The ship detection data will be compared to the collected AIS data, highlighting ships that are not transmitting AIS. 
Figure 3 reflects the sequential workflow of additional features for the maritime monitoring and surveillance system and an automatic ship detection algorithm. These are pipelined for integration on the SIYASAT Portal so that the results generated from the system's modules are available for viewing online.

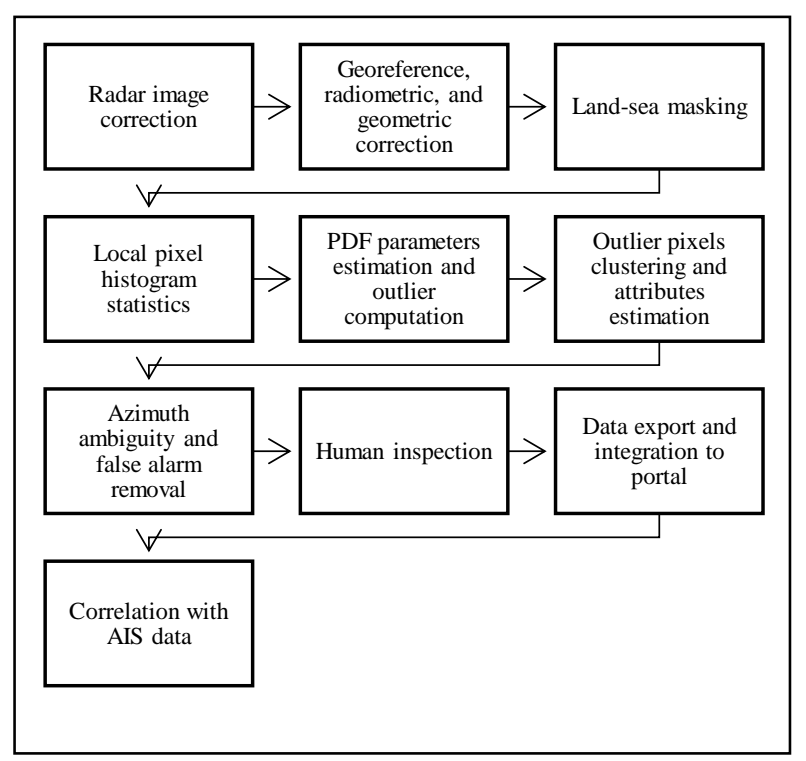

Figure 3. Generated workflow for the maritime monitoring and surveillance system and automatic ship detection algorithm.

Level 1 images are georeferenced with GPS data with calibrated backscatter values. To narrow down the search area, sea masks are applied using the Philippines' Exclusive Economic Zone (EEZ) with a coastline buffer of 3 kilometers to address subtle georeferencing errors. Constant false alarm rate detectors aim to identify pixels that are potential outliers with respect to its neighbors (Serco, 2018). The algorithm assumes a Gaussian probability distribution function and determines an outlier if the pixel value exceeds the adaptive threshold set by the false alarm rate. Adjacent outlier pixels are clustered and their sizes are estimated. Pixel groups that fit the target size criteria are regarded as ships and others are discarded since they are false alarms.

SNAP software was used in reading and processing NovaSAR images with its graph processing tool for automating ocean object detection. Target window, guard window and background window sizes used to isolate outliers were set to 50,500 and 800 meters. The false alarm rate, or the probability of false detection, was set to $6.5 \%$, which means that an outlier pixel value must belong to the top $6.5 \%$ of the histogram.

For object discrimination, minimum and maximum target sizes were set to 50 and 600 meters respectively. Figure 4 shows potential ships detected from a 20-meter ScanSAR image taken over the West Philippine Sea.

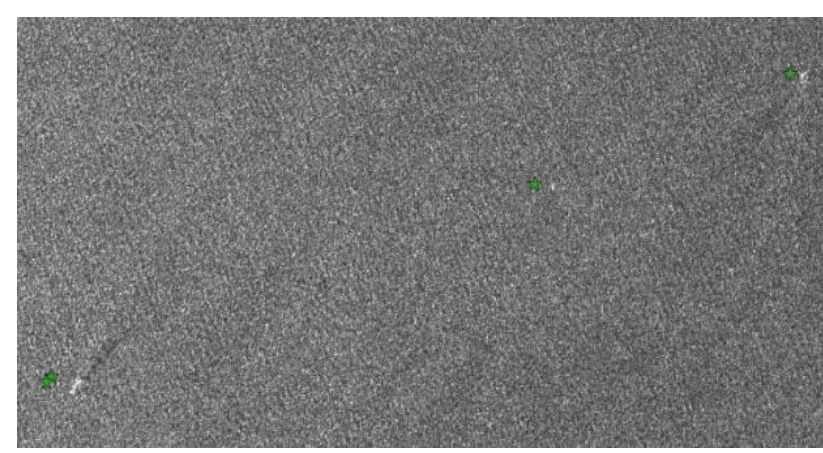

Figure 4. Ship detection results on a NovaSAR-1 20-m ScanSAR image (subset)

The test image has a swath of $23 \mathrm{~km}$ by $2,900 \mathrm{~km}$ with a size of 3.5GB. Ship positions were also adjusted relative to the satellite's current position and velocity. All potential ships seen by the human eye have been detected since the image is in a calm sea state. More targets were captured with lower false alarm rate and smaller target size, but these are prone to detecting false positives such as random speckles. Fine-tuning the parameters is required to maximize true positives while minimizing false positives while considering image capture mode used and conditions of the sea.

Other methods of ship detection are also in the process of development to consider the shape of the object aside from its pixel values. These include object-based image analysis with support vector machines and image segmentation using convolutional neural networks. Detection accuracy, time and space requirements will be compared for large-scale operational use on different computing facilities in the country.

2.4 Future Applications. Ships that do not transmit AIS signals will be logged within the system to form a database for the government to police erring vessels. A geofencing feature will also be developed to alert monitoring agencies once a vesse is detected in a marine protected area, either through AIS or the automatic ship detection algorithm.

\section{CONCLUSION}

This paper provides an insight of the Philippines' ongoing formulation, development, and implementation of an automated maritime surveillance application and ship detection algorithm, through the DOST-ASTI. The implementation of the project, along with the participation of its partners and relevant agencies, relies on the use EO and SAR remote sensing data and its subsequent analysis.

Looking forward, the possibilities of system integration with more data sources and higher-end facilities and exploring further points of analysis for SAR with AIS data is on the line for the project. Horizontal and vertical linkages with more stakeholders should be introduced to widely utilize and operationalize the availability of raw and processed SAR and AIS data. The discussed applications can contribute to the achievement of Targets 14.5 and 14.6, with relevant interests on other Goal 14 targets such as 14.2, 14.4, and 14.C. Further, the deployment of 
the project helps strengthen the Philippines' management over its maritime assets.

Additional study needs to be done to assess the current situation and identify research needs in the context of each sector to count progress towards the SDG, as the accomplishment of Goal 14's targets through its indicators requires full integration of policies, the government, communities, and as evidenced by scientific research and output.

\section{ACKNOWLEDGEMENTS}

The overall contributions of the team behind the SAR with AIS project is invaluable. The implementation of the SAR with AIS project is made possible by the Department of Science and Technology (DOST) and the DOST-Philippine Council for Industry, Energy, and Emerging Technology Research and Development (PCIEERD) through its Grants-in-Aid (GIA) Program, the National Security Council (NSC) and the National Coast Watch System (NCWS).

\section{REFERENCES}

Achiri, L., Guida, R., and Iervolino, P. 2018. Collaborative use of SAR and AIS data from NovaSAR-S for Maritime Surveillance. 12th European Conference on Synthetic Aperture Radar (EUSAR 2018), 04-06 Jun 2018, Aachen, Germany.

Anderson, K., Ryan, B., Sonntag, W., Kavvada, A., and Friedl, L. 2017. Earth observation in service of the 2030 Agenda for Sustainable Development. Geo-spatial Information Science 20(2), p. 77-96. DOI: 10.1080/10095020.2017.1333230.

Chaturvedi, S., Yang, C., Ouchi, K., and Shanmugam, P. 2012. Ship recognition by integration of SAR and AIS. The Journal Of Navigation (2012), 65, p. 323-337. The Royal Institute of Navigation. DOI: 10.1017/S0373463311000749.

Meyer, F. and Hinz, S. 2009. Automatic ship detection in spaceborne SAR images. International Society for Photogrammetry and Remote Sensing (ISPRS) Conference Proceedings, Volume XXXVIII-1-4-7/W5, 2009.

F. Papi, D. Tarchi, M. Vespe, F. Oliveri, F. Borghese, G. Aulicino, A Vollero. 2014. Radiolocation of Automatic Identification System Signals for Maritime Situational Awareness. IET Radar, Sonar \& Navigation, Volume 9 Issue 5. 10.1049/iet-rsn.2014.0292.

Garcia, M. 2015. Progress in the Implementation of the Philippine National Marine Policy: Issues and Option. https://www.un.org/depts/los/nippon/unnff_programme_home/f ellows_pages/fellows_papers/garcia_0506_philippines.pdf

Pichel, W., Clemente-Colón, P., Wackerman, C., Friedman, K. 2018. Chapter 12. Ship and Wake Detection. In Synthetic Aperture Radar Marine User's Manual. National Oceanic and Atmospheric Administration, US Department of Commerce, Camp Springs, Maryland, USA.
Prado, N. M., Judan, J. M., Tabanggay, L. A., Vicente, R. B., Paler, H. S., and Retamar, A. E. 2019. The Philippine Earth Data Resource and Observation Center - An Enabler in Disaster Risk Reduction and Management. Paper presented at the Asian Conference on Remote Sensing 2019, Daejeon, South Korea, 1318 October 2019.

Sturesson, A., Weitz, N. and Persson, A. 2018. SDG 14: Life Below Water. A Review of Research Needs. Technical annex to the Formas report Forskning för Agenda 2030: Översikt av forskningsbehov och vägar framåt. Stockholm Environment Institute, Stockholm.

Virto, L. 2018. A preliminary assessment off the indicators for Sustainable Development Goal (SDG) 14 "Conserve and sustainability use the oceans, seas and marine resources for sustainable development." Marine Policy 98 https://doi.org/10.1016/j.marpol.2018.08.036

Palomino, E., 2020. SDG 14 Life Below Water 229-246. https://doi.org/10.1007/978-981-32-9927-6_15

United Nations (UN), 2017. Life Below Water: Why it Matters. https://doi.org/10.18356/85817b57-en

United Nations Office for Outer Space Affairs (UNOOSA) 2020. Sustainable Development Goal 14: Life Below Water. Retrieved from: https://www.unoosa.org/oosa/en/ourwork/space4sdgs/sdg14.ht $\mathrm{ml}$

United States Department of Homeland Security, United States Coast Guard. n.d. AIS Messages. Retrieved from: https://www.navcen.uscg.gov/?pageName=AISMessages

exactEarth Ltd. Satellite AIS and First Pass Detection. 2018. Retrieved from: http://cdn2.hubspot.net/hub/183611/file30951507-

pdf/Collateral_for_Download/First_Pass_Detection_White_Pap er.pdf

Serco Italia SPA. 2018. Ship detection with Sentinel-1 - Gulf of Trieste (version 1.3). Retrieved from: RUS Lectures at https://rus-copernicus.eu/portal/the-rus-library/learn-byyourself/ 